\title{
The reliability of isoinertial force-velocity-power profiling and maximal strength assessment in youth
}

\author{
CÉSAR M.P. MEYLAN ${ }^{1,2,3}$, JOHN B. CRONIN ${ }^{1}$, JON L. OLIVER ${ }^{4}$, MICHAEL M.G. HUGHES ${ }^{4}$, BORIS \\ JIDOVTSEFF $^{5}$, \& SHANE PINDER ${ }^{1}$ \\ ${ }^{1}$ Sport Performance Research Institute New Zealand, AUT University, Auckland, New Zealand, ${ }^{2}$ Canadian Sport \\ Institute Pacific, Vancouver, BG, Canada, ${ }^{3}$ Canadian Soccer Association, Ottawa, ON, Canada, ${ }^{4}$ Cardiff School \\ of Sport, Cardiff Metropolitan University, Cardiff, Wales, UK, and ${ }^{5}$ Department of Sports Sciences, Service of \\ Training and Assessment of Physical Fitness, University of Liege, Liege, Belgium
}

\begin{abstract}
The purpose of this study was to quantify the inter-session reliability of force-velocity-power profiling and estimated maximal strength in youth. Thirty-six males (11-15 years old) performed a ballistic supine leg press test at five randomized loads $(80 \%, 100 \%, 120 \%, 140 \%$, and $160 \%$ body mass) on three separate occasions. Peak and mean force, power, velocity, and peak displacement were collected with a linear position transducer attached to the weight stack. Mean values at each load were used to calculate different regression lines and estimate maximal strength, force, velocity, and power. All variables were found reliable (change in the mean $[\mathrm{CIM}]=-1$ to $14 \%$; coefficient of variation $[\mathrm{CV}]=3-18 \%$; intraclass correlation coefficient $[$ ICC $]=0.74-0.99)$, but were likely to benefit from a familiarization, apart from the unreliable maximal force/velocity ratio (CIM = $0-3 \%$; CV $=23-25 \%$; ICC $=0.35-0.54)$ and load at maximal power $(\mathrm{CIM}=-1$ to $2 \%$; $\mathrm{CV}=10-13 \%$; ICC $=0.26-$ 0.61). Isoinertial force-velocity-power profiling and maximal strength in youth can be assessed after a familiarization session. Such profiling may provide valuable insight into neuromuscular capabilities during growth and maturation and may be used to monitor specific training adaptations.
\end{abstract}

KEYWORDS: Jumping, force measurement, kinetics, special population

\section{INTRODUCTION}

Maximal power output is the product of force and velocity and is defined and limited by the force-velocity relationship (Fenn \& Marsh, 1935; Hill, 1938). On this basis, maximal power output may improve by increasing the ability to develop high levels of force at a given velocity (i.e. force capability or strength) and/or higher velocity at a given force (i.e. velocity capability) (Cormie, McGuigan, \& Newton, 2011). Assessing the isoinertial force-velocity-power relationship has previously been found valuable to assist in understanding the underlying mechanisms responsible for maximal power output (Rahmani, Viale, Dalleau, \& Lacour, 2001; Samozino, Rejc, Di Prampero, Belli, \& Morin, 2012) and training adaptations (Cormie, McGuigan, \& Newton, 2010a, 2010b). The vertical jump is a commonly used movement to assess isoinertial leg power because of its simplicity and explosiveness (Cormie et al., 2011). The kinematics and kinetics of unloaded and loaded jump squats have been found reliable and used to determine the force-velocity-power profiling of the lower limbs neuromuscular system in adults (Cormie et al., 2010a, 2010b; Sheppard, Cormack, Taylor, McGuigan, \& Newton, 2008). Previous studies have also used the load-power relationship during vertical jump to determine the 'optimal' load for maximal power in adults (Cormie et al., 2011) and athletic children and adolescents (Dayne et al., 2011; Viitasalo, 1988; Viitasalo, Rahkila, Osterback, \& Alen, 1992). However, the variability of the forcevelocity-power relationship of an isoinertial loaded protocol in youth is unknown. Monitoring mechanistic adaptations may provide better training information than a single point on a curve (e.g. peak power) since previous studies have demonstrated that profiles may be force or velocity dominant (Samozino et al., 2012) and influenced by the training mode (Cormie et al., 2010a). As considerable changes associated with growth and maturation are likely to influence force, velocity, and power output during development (e.g. muscle crosssectional area, fascicle length) (Van Praagh \& Dore, 2002), athlete mechanistic profiling from a young age would enhance long-term athlete development.

Maximal strength as measured by a one repetition maximum (1RM) is a functional measure of neuromuscular force capability and is usually determined by lifting maximal loads. Recently maximal strength has been predicted based on the load-velocity relationship using a loading protocol (Jidovtseff, Harris, Crielaard, \& 
Cronin, 2011). The obvious benefits of this estimation are that submaximal lifting is considered safer for athletes with little weightlifting history such as children and adolescents and the reduction of testing time to reach one maximal lift (7-11 trials) (Faigenbaum, Milliken, \& Wescott, 2004) while also assessing the force-velocitypower profile of young athletes (Jidovtseff et al., 2011). However, the prediction of maximal strength from the load-velocity relationship was undertaken in an adult population and whether maximal strength can be accurately and reliably established in a youth population is unknown.

Using loaded jumps may not be advisable for general paediatric populations due to poor squatting and landing technique, overloading of the growing spine, and increased force during landing (Faigenbaum et al., 2009). Force-velocity-power profiles of children and adolescents in cycling were found reliable (Dore et al., 2003; Santos, Welsman, De Ste Croix, \& Armstrong, 2002), but the validity of such tests for field sport athletes is questionable (Van Praagh, 2008) and does not allow for estimation of maximal strength. Previous studies have demonstrated the safety of ballistic movement on a supine squat machine with novice and experienced weightlifters (Cronin \& Henderson, 2004; Samozino et al., 2012). As this movement simulates jump mechanics, it could be used to determine the isoinertial force-velocity-power profile of youth athletes. Inertial dynamometers (e.g. linear position transducers or accelerometers) have been found to be reliable in combination with this equipment and associated methodologies (Cronin \& Henderson, 2004) and have been used with adults to create load-velocity (Jidovtseff et al., 2011) and load-power profiles (Cronin, Jones, \& Hagstrom, 2007; Harris, Cronin, \& Hopkins, 2007). However, this methodology is yet to be applied to youth population. Such analysis would provide a safe method to better understand mechanistic changes during growth, and the influence of maturation on force-velocity-power relationship and profile.

Establishing the reliability of new assessments in novel populations, such as children, is critical to determine the ability of the test to monitor changes in athletic performance over time (Hopkins, 2000). The variation in performance can be attributed to biological sources (e.g. changes in an individual jump height between trials because of changes in physical state) or equipment. Establishing the typical variation of performance measures assists coaches and sports scientists in confidently assessing the effects of training interventions by taking into account the changes that are likely due to the 'noise' involved in the testing methods as well as typical daily biological variation present within a group of athletes. Hopkins (2000) recommended that a systematic change in mean (CIM) as well as measures of absolute and relative consistency (i.e. within-subject variation and retest correlations respectively) be reported to gain a true appreciation of the reliability of measures. Given this information, the purpose of this study was to quantify the inter-session reliability of force-velocity-power profiling in youth using a ballistic loading protocol on a supine leg press. It is hypothesized that the ballistic loading protocol would be reliable in measuring force, velocity and power in youth, and the maximum power and associated optimal load and velocity, predicted $1 \mathrm{RM}$, as well as maximal force and velocity could be predicted by a regression analysis.

\section{METHODS}

\section{Participants}

Thirty-six youth males between 11 and 15 years of age volunteered for this study (Table I). All participants were nominated by their physical education teacher to be part of a school sports academy. The Human Research Ethics Committee of AUT University approved the study and both the participants and their parents/guardians gave their written consent/assent prior to the start of the study.

\section{Testing procedures}

Anthropometric measurements were taken before the first performance testing on the supine squat machine. The standing height $(\mathrm{cm})$, sitting height $(\mathrm{cm})$ and body mass $(\mathrm{kg})$ were measured and the body mass index (BMI) was calculated. The maturity status of the athletes was determined using years from peak height velocity (i.e. PHV offset) (Mirwald, Baxter-Jones, Bailey, \& Beunen, 2002) as well as the percentage of predicted adult stature (PAS) (Khamis \& Roche, 1994).

Participants attended three designated testing sessions at the same time of the day separated by 7 days. Prior to testing, participants undertook a 15-min standardized warm-up using the different loads employed in the testing. Performance testing consisted of three trials of ballistic concentric squats on a supine squat machine (Fitness Works, Auckland, New Zealand) at five different relative loads (80\%, 100\%, 120\%, 140\%, and 160\% body mass) performed in a randomized order. Prior to each load, participants were asked to fully extend their leg to determine the zero position, which was used to determine the end of the pushing phase. A recovery of $30 \mathrm{~s}$ 
between trials within load and $120 \mathrm{~s}$ between loads was given. The sled lay on top of an undercarriage, which enabled the sled to be pegged every $2 \mathrm{~cm}$, allowing standardization of the foot position and knee angle $\left(70^{\circ}\right)$ using a shin position parallel to the ground and goniometer, respectively (Cronin \& Henderson, 2004). The supine squat machine was designed to allow novice participants to perform maximal squats or explosive squat jumps, with the back rigidly supported, thus minimizing the risk associated with such exercises in an upright position (e.g. excessive landing forces, lumbar spine flexion and extension) (Figure 1).

Table I. Subjects characteristics.

\begin{tabular}{ll}
\hline Variables & Mean $\pm S D$ \\
\hline Age (year) & $13.1 \pm 1.1$ \\
Height $(\mathrm{cm})$ & $160 \pm 10.6$ \\
Mass $(\mathrm{kg})$ & $49.5 \pm 11.6$ \\
Leg length $(\mathrm{cm})$ & $77.3 \pm 5.5$ \\
Peak height velocity offset (year) & $-0.7 \pm 1.2$ \\
PAS $(\%)$ & $89.6 \pm 5.9$ \\
BMI $\left(\mathrm{kg} / \mathrm{m}^{2}\right)$ & $19.0 \pm 2.7$ \\
Body fat $(\%)$ & $14.8 \pm 4.8$ \\
\hline
\end{tabular}

A linear position transducer (Model PT9510-0150-112-1310, Celesco, Chatsworth, USA) attached to the weight stack measured vertical displacement relative to the ground with an accuracy of $0.1 \mathrm{~cm}$. These data were sampled at $1,000 \mathrm{~Hz}$ by a computer-based data acquisition and analysis program. The raw displacement-time data were filtered before differentiation using a low-pass 4th-order Butterworth filter with a cut-off frequency of $50 \mathrm{~Hz}$, to obtain position and remove non-physical electrical noise from the signal. The cutoff frequency of 50 $\mathrm{Hz}$ is the main power frequency in New Zealand, where the tests were performed, and the characteristic attenuation of signals above the cut-off frequency in this filter were sufficient to remove this electrical noise. The filtered position data were then differentiated using the finite-difference technique to determine velocity and acceleration data of the weight stack, which were each successively filtered using a low-pass 4th-order Butterworth filter with a cut-off frequency of $6 \mathrm{~Hz}$ (Harris et al., 2007). The cut-off frequency of $6 \mathrm{~Hz}$ is high relative to the physical dynamics of a ballistic thrust, and therefore no physically indicative information was lost using this cut-off frequency. The instantaneous vertical force $(F)$ produced during the thrust was determined by adding the weight of the weight stack to the force required to accelerate the system mass, which consisted of the mass of the weight stack $\left(\mathrm{m}_{\mathrm{WS}}\right)$ the mass of the participant $\left(\mathrm{m}_{\mathrm{P}}\right)$, and the mass of the sled $\left(\mathrm{m}_{\mathrm{S}}\right)$

$$
F=g\left(m_{w s}\right)+a\left(m_{w s}+m_{F}+m_{s}\right),
$$

where $g$ is the acceleration due to gravity and $a$ is the acceleration generated by the movement of the participant. Following these calculations, power was determined by multiplying the force by velocity at each time point. Average force, velocity and power were determined from the averages of the instantaneous values over the entire push-off phase (until full leg extension, i.e. position 0 ). The external validity of the derived measurements from a linear position transducer have been demonstrated using the force plate as a 'gold standard' device $(r=0.81-0.96)$ (Cronin, Hing, \& McNair, 2004; Harris, Cronin, Taylor, \& Jidovtseff, 2010; Hori et al., 2007), but the linear position transducer has been reported to underestimate force and power output in comparison to force plate data (Hori et al., 2007). 
Figure 1. Experimental set-up on the supine squat machine. The linear position transducer was attached to the weight stack to provide displacement data output as the participant moved horizontally.

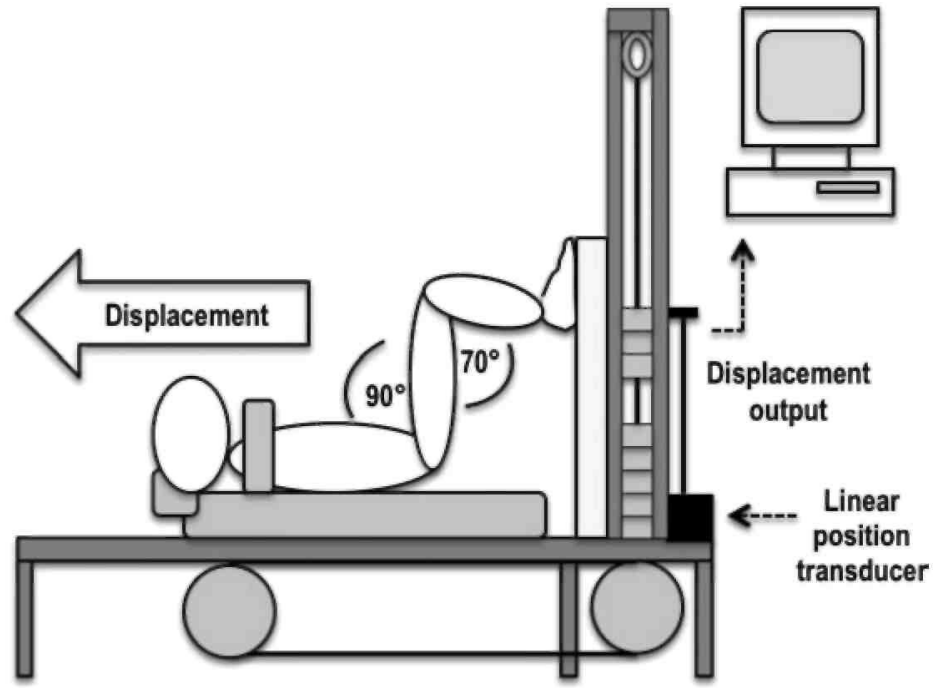

\section{Data analysis}

1RM was estimated via the load-velocity relationship (Jidovtseff et al., 2011). The 1RM velocity was not calculated in the current study and this value $(0.23 \mathrm{~m} / \mathrm{s}$ ) was extracted from previous studies in adults (Harris et al., 2007; Jidovtseff et al., 2011) to be plotted on the load-velocity curve to extract 1RM (Figure 2). A pilot study on 10 children involved in the current study found a 5\% (confidence limits [CL]: 9.5-0.2\%) underestimation of the actual $1 \mathrm{RM}(118.5 \pm 27.3 \mathrm{~kg})$ when compared to the predicted $1 \mathrm{RM}$ at $0.23 \mathrm{~m} / \mathrm{s}(112.1 \pm 23.0 \mathrm{~kg})$. Pearson's correlation of 0.94 (CL: 0.80-0.98) between the two measures was similar to the findings reported by Jidovtseff et al. $(\mathrm{r}=0.95-0.96)$ (Jidovtseff et al., 2011).

Force-velocity relationships were determined by least-squares linear regressions using average force and velocity at each load. Individual force-velocity slopes were extrapolated to obtain maximal force and velocity, which corresponded to the intercepts of the force-velocity slope with the force and velocity axes, respectively (Rahmani et al., 2001; Samozino et al., 2012) (Figure 3). Since the power-load and power-velocity relationship is derived from the product of force and velocity, it was logically described by second-degree polynomial functions and maximal power output and the optimal load and velocity at which maximal power output occurred was determined using the power-load and power-velocity regression curves, respectively (Harris et al., 2007). The goodness-of-fit of the individuals' quadratics was expressed as a correlation coefficient calculated by taking the square root of the fraction of the variance explained by the model, after adjusting for degrees of freedom; the values were then averaged. The curve was also used to estimate the percent decline in maximal power at loads of $10 \%$ and $20 \%$ of body mass either side of the maximum power output. 
Figure 2. Average velocity-relative load (\% body mass) relationship. The dynamic one repetition maximum $(1 R M$ at $0.23 \mathrm{~m} / \mathrm{s})$, isometric $1 R M(1 R M$ at $0 \mathrm{~m} / \mathrm{s})$, and maximal velocity ( $V_{\text {max }}$ were derived from the loadvelocity slope of the mean values between sessions 2 and 3 observed in the whole sample. The relationship between the two 1RM was $r=0.99$.

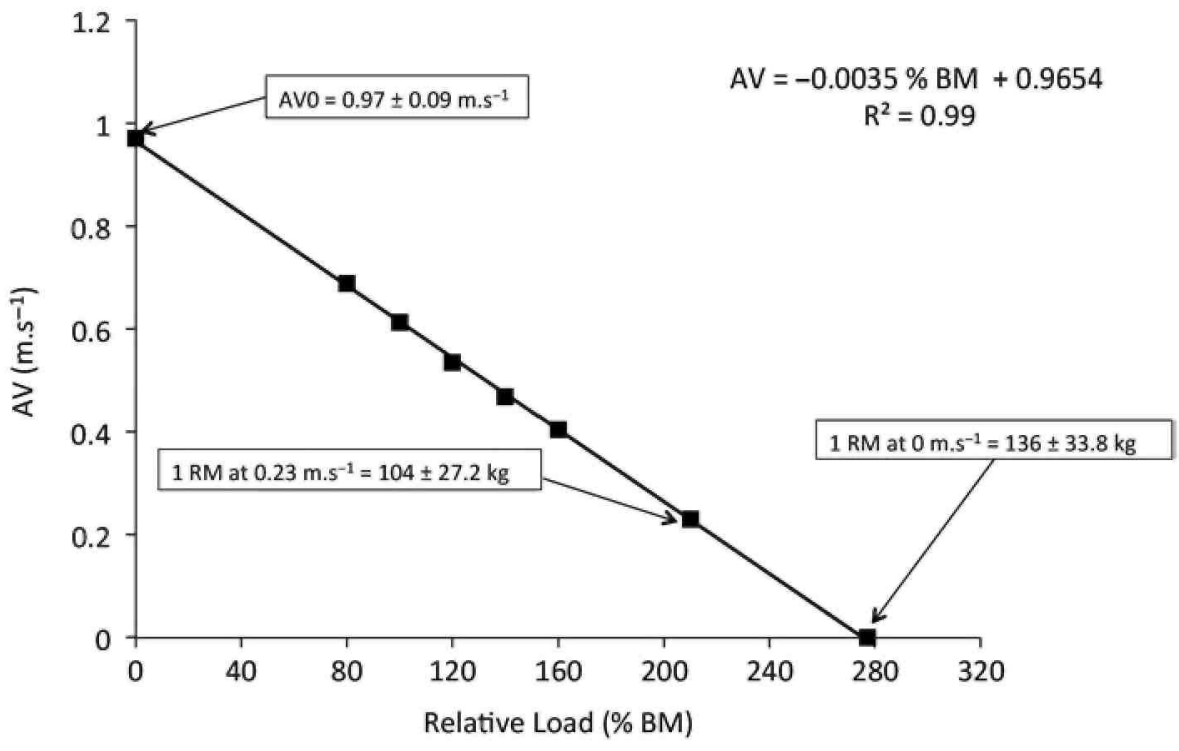

\section{Statistical analysis}

Following data collection, means and standard deviations were calculated for all variables of interest. The three trials for all ballistic squat variables at different loads were averaged for an individual subject mean for each session. To determine the influence of maturity on all dependent variables, initial data analysis was conducted on the individual inter-session coefficient of variation $(\mathrm{CV})$ in all loads. The sample was split into three groups: post-growth spurt (+1.37 to +0.05 years from PHV and 97.7-90.7\% PAS; $n=13)$; in-growth spurt $(-0.01$ to -1.45 years from PHV and 92.6-85.2\% PAS; $n=14)$; and pre-growth spurt $(-1.66$ to -2.67 years from PHV and 85.2$80.9 \%$ PAS; $n=9$ ). A one-way analysis of variance (ANOVA) on the CV was conducted to determine any significant difference in reliability across the three groups. Assumptions of normality and homoscedasticity were checked with Shapiro-Wilk and Levene tests, respectively. Since there was no significant difference amongst the groups $(p>0.05)$, the effect of maturity on reliability was deemed negligible and the data were pooled for further analysis.

The inter-session reliability was calculated using three different statistical methods using pairwise comparisons with $90 \%$ CL with log-transformed data to reduce the effects of any non-uniformity of error (Hopkins, 2000). The standard error of measurement, expressed as a $\mathrm{CV}$, was reported to determine the absolute reliability or within subject variation of the different variables (Hopkins, 2000). Percent CIM was reported to indicate the extent to which the average performance changed over testing occasion due to systematic effects (e.g. learning effect) and random effect (e.g. noise) (Hopkins, 2000) and relative reliability was quantified via an intraclass correlation coefficient (ICC). The level of acceptance for reliability was a CV $\leq 15 \%$ (Stokes, 1985) and ICC $\geq$ 0.70 (Vincent, 1994). Reliability comparison of the dependent variables from sessions 1-2 to sessions 2-3 was investigated by calculating the confidence intervals for the ratio of the $\mathrm{CV}$ between sessions, using the fact that the sampling distribution of the ratio of the sample to population variances in the two groups is an F-distribution. We regarded a CV that differed by a factor of 1.15 or more as being substantially different, because the effect of such a difference on sample size in a controlled trial of competitive performance is a factor of 1.15 (Hopkins \& Hewson, 2001), or a change in sample size of 32\%. The chance of 'beneficial/better' or 'detrimental/poorer' reliability from session to session was assessed qualitatively as follows: possible (25-75\%), likely (75-95\%), very likely $(95-99.5 \%)$, and most likely $(>99.5 \%)$. If the probabilities of the effect being substantially positive and negative were both $>5 \%$, the effect was reported as unclear (Hopkins, Marshall, Batterham, \& Hanin, 2009). 
Figure 3. Illustration of force-velocity (left panel) and power-velocity (right panel) relationships for two subjects with different force-velocity profiles and maximal power values (grey cross). Participant 1 (open squares) presents a lower maximal power and a force-velocity profile more oriented towards velocity capabilities than participant 2 (filled squares), who presents a force-velocity profile more oriented towards force capabilities.
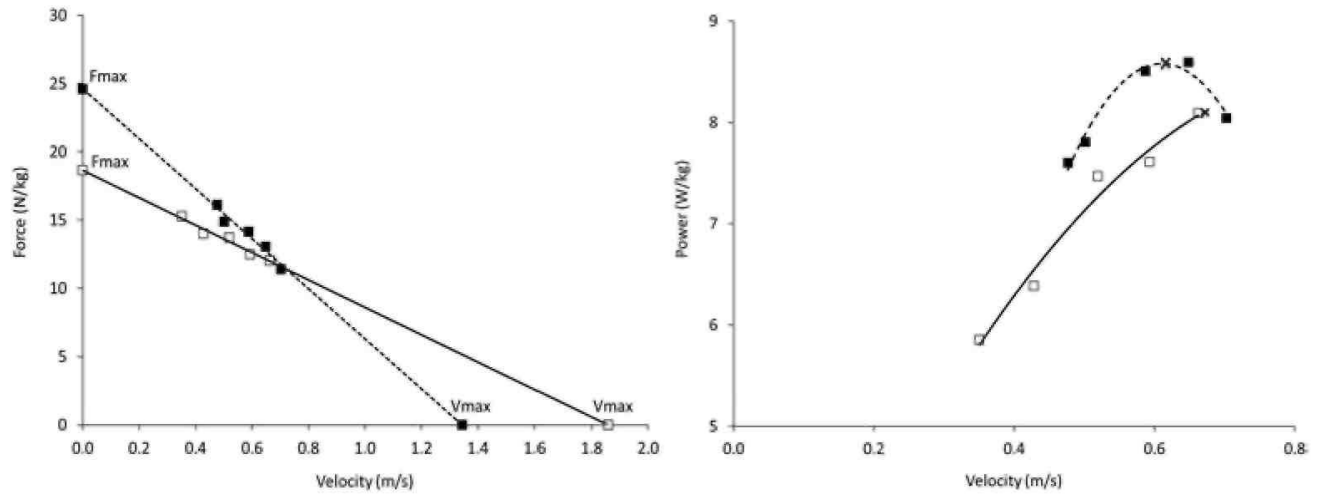

\section{RESULTS}

The mean and standard deviation of the kinetics and kinematics across the load of testing session 1 are presented in Table II. Peak and mean force increased with increasing load, whereas velocity and subsequent displacement decreased. Most of the kinetic and kinematic variables of interest (see Table III) were found reliable across multiple testing occasions for the different loads $(\mathrm{CIM}=1-14 \% ; \mathrm{CV}=3-18 \%$; ICC $=0.74-0.99)$. Peak and mean force were the most reliable while peak displacement was the most variable. After comparing CV's between sessions 1-2 and sessions 2-3, it was found that $60 \%$ of the variables were classified as likely, very likely or most likely to be more reliable from sessions 2 to 3 compared to sessions $1-2$. The loads at $80 \%$ and $160 \%$ body mass were the most likely to benefit from a third session, with six and four from seven variables improving substantially between sessions 2 and 3 ( $>75 \%$ of a beneficial outcome).

Mean, standard deviation, and reliability statistics of 1RM, maximal force, maximal velocity, maximal force/velocity slope; maximal power (at optimal load and optimal velocity), optimal load, and optimal velocity estimations are presented in Table IV. Based on the strong load-velocity relationship (mean goodness-of-fit session 2- $3 R^{2}=0.99$ ) (see Figure 2), 1RM was found reliable and the effect of familiarization unclear (see Table IV). Maximal force and maximal velocity, which were derived from the force-velocity relationship (mean goodness-of-fit third session $\left.R^{2}=0.90\right)$, were found to be relatively reliable (CIM = 0.8-6.9; CV=7.2-12.4; ICC $=0.57-0.80)$. Maximal velocity was likely to benefit from a familiarization session and the maximal force/velocity slope was found unreliable (see Table IV).

Table II. Measurements of session 1 (Mean \pm SD) across different loads.

\begin{tabular}{llllll}
\hline & \multicolumn{5}{c}{ Load (\% body mass) } \\
\cline { 2 - 6 } Variables & 80 & 100 & 120 & 140 & 160 \\
\hline Peak force (N) & $678 \pm 209$ & $744 \pm 213$ & $797 \pm 229$ & $880 \pm 245$ & $940 \pm 261$ \\
Mean force (N) & $539 \pm 161$ & $595 \pm 164$ & $635 \pm 171$ & $691 \pm 175$ & $737 \pm 190.6$ \\
Peak velocity (m/s) & $1.21 \pm 0.17$ & $1.12 \pm 0.15$ & $1.00 \pm 0.14$ & $0.91 \pm 0.14$ & $0.83 \pm 0.15$ \\
Mean velocity (m/s) & $0.64 \pm 0.08$ & $0.57 \pm 0.08$ & $0.50 \pm 0.08$ & $0.42 \pm 0.08$ & $0.37 \pm 0.09$ \\
Peak power (W) & $713 \pm 300$ & $735 \pm 285$ & $714 \pm 284$ & $712 \pm 279$ & $710 \pm 291$ \\
Mean power (W) & $362 \pm 149$ & $362 \pm 138$ & $339 \pm 133$ & $315 \pm 116$ & $297 \pm 126$ \\
Peak displacement (cm) & $27.2 \pm 6.8$ & $21.8 \pm 5.1$ & $16.9 \pm 4.4$ & $14.2 \pm 3.8$ & $11.9 \pm 3.9$ \\
\hline
\end{tabular}

Note: All variables are in the vertical direction. 
Table III. Range in reliability statistics for the direct variables of interest from supine squat jump across the five loads.

\begin{tabular}{|c|c|c|c|c|c|c|}
\hline \multirow[b]{2}{*}{ Variables } & \multicolumn{2}{|c|}{ Change in the mean ${ }^{\mathrm{a}}(\%)$} & \multicolumn{2}{|c|}{ Error of measurement ${ }^{\mathrm{b}}(\mathrm{CV} \%)$} & \multicolumn{2}{|c|}{ Intraclass correlation $^{c}$} \\
\hline & $\begin{array}{l}\text { Sessions } \\
1-2\end{array}$ & Sessions 2-3 & Sessions 1-2 & Sessions 2-3 & Sessions 1-2 & Sessions 2-3 \\
\hline Peak force & $3.4-5.1$ & $1.8-2.4$ & $4.3-5.4$ & $3.0-4.3$ & $0.97-0.98$ & 0.98-0.99 \\
\hline Mean force & $2.8-5.4$ & $0.5-2.6$ & $3.2-4.7$ & $2.6-4.0$ & $0.98-0.99$ & $0.98-0.99$ \\
\hline Peak velocity & $4.1-6.0$ & $1.7-3.6$ & 5.3-8.2 & $3.1-5.5$ & $0.81-0.86$ & $0.88-0.94$ \\
\hline Mean velocity & $6.4-12$ & $1.3-3.4$ & $4.6-9.9$ & $5.0-8.4$ & $0.80-0.87$ & $0.77-0.89$ \\
\hline Peak power & $6.9-9.4$ & $2.7-4.9$ & $10-12$ & $5.7-8.7$ & $0.93-0.94$ & $0.96-0.98$ \\
\hline Mean power & $11-14$ & $3.1-6.2$ & $8.6-13$ & $6.6-11$ & $0.92-0.95$ & $0.92-0.96$ \\
\hline $\begin{array}{l}\text { Peak } \\
\text { displacement }\end{array}$ & $1.6-11$ & $0.4-8.2$ & $12-18$ & $5.5-12$ & $0.74-0.86$ & $0.84-0.94$ \\
\hline
\end{tabular}

Note: All variables are in the vertical direction.

a $90 \%$ Confidence limits: $\pm 0.40 \times$ error of measurement $= \pm$ (error of measurement $) \times(\sqrt{ } 2 / \sqrt{ } \mathrm{n}) \times t$; where $n$ is the sample size, and $t$ is the $t$ statistic for $90 \%$ CL.

b $90 \%$ CL: $\div / \times 1.20$

${ }^{\mathrm{c}} 90 \% \mathrm{CL}:$ lowest $\mathrm{ICC} \pm 0.13$, highest $\mathrm{ICC} \pm 0.01$

The reliability of maximal power at optimal load and optimal velocity were acceptable $(\mathrm{CIM}=4-14 \%$; CV $=6$ $12 \%$; ICC $=0.91-0.97$ ) but maximal power at optimal velocity was very likely to benefit from a familiarization session. Maximal power at optimal velocity and maximal power at optimal load were found to be significantly correlated $(\mathrm{r}=0.98$; CL: 0.96-0.99). However, only optimal velocity was deemed reliable but was also very likely to benefit from a familiarization session. The mean goodness-of-fit of the power-load and power-velocity curve were $R^{2}=0.81$ and 0.82 , respectively.

An estimated load of $87.6 \%$ body mass ( $\pm 14.6 \%$ - session 1$)$ was found to maximize power output. Individual maximal power outputs ranged from $80 \%$ to $129 \%$ body mass, but $50 \%$ of the participants had a theoretical optimal load less than $80 \%$ body mass. Figure 3 highlights the difference between two participants with different force-velocity and power-velocity relationships. A $10 \%$ and $20 \%$ change in load each side of this maximum resulted in a $2.2 \%$ and $5.5 \%$ decrease in power output, respectively.

\section{DISCUSSION AND IMPLICATIONS}

The aim of the current study was to determine the variability of a new isoinertial protocol to assess forcevelocity-power and maximal strength in a paediatric population. The intersession reliability of the kinematics and kinetics from the isoinertial loaded ballistic supine squats was deemed acceptable. Similar reliability statistics (CIM $=-1$ to $5 \%$; $\mathrm{CV}=3-10 \%$; ICC $=0.71-0.95)$ have been reported in research with adults (Sheppard et al., 2008). Previous studies in youth have shown that the jump height variability increased during an incremental loading protocol from unloaded to $40 \mathrm{~kg}$ (Viitasalo, 1988). This trend was echoed in the current study, as poorer yet acceptable reliability was found at the $160 \%$ body mass load and the most reliable data were recorded at body mass. However, when the load became lower than the body mass (80\%), the reliability was also affected. It can be concluded that increased biological variability in young athletes occurred when movement velocity or force requirements were high and influenced by learning. It is recommended thatan in-depth familiarization across the load spectrum should be conducted, after which force-velocity-power profiling in youth can be confidently implemented to monitor mechanistic determinants of movement during growth and maturation. 
Table IV. Session 1 derived variables measurement $(M \pm S D)$ and reliability across the three sessions based on the incremental loading protocol.

\begin{tabular}{|c|c|c|c|c|c|c|c|}
\hline \multirow[b]{2}{*}{ Variables } & \multirow{2}{*}{\begin{tabular}{|l} 
Mean $\pm S D$ \\
Session 1
\end{tabular}} & \multicolumn{2}{|c|}{$\begin{array}{l}\text { Change in the mean }{ }^{\mathrm{a}} \\
(\%)\end{array}$} & \multicolumn{2}{|c|}{$\begin{array}{l}\text { Standard error of } \\
\text { mean }^{b}(\mathrm{CV} \%)\end{array}$} & \multicolumn{2}{|c|}{ Intraclass correlation $^{\mathrm{c}}$} \\
\hline & & \begin{tabular}{|l} 
Sessions \\
$1-2$ \\
\end{tabular} & $\begin{array}{l}\text { Sessions } \\
2-3\end{array}$ & $\begin{array}{l}\text { Sessions } \\
1-2 \\
\end{array}$ & $\begin{array}{l}\text { Sessions } \\
2-3 \\
\end{array}$ & \begin{tabular}{|l} 
Sessions \\
$1-2$ \\
\end{tabular} & $\begin{array}{l}\text { Sessions 2- } \\
3\end{array}$ \\
\hline 1RM (kg) & $98.1 \pm 25.4$ & 5.1 & 1.6 & 6.0 & 6.8 & 0.95 & 0.94 \\
\hline $\mathrm{F}_{\max }(\mathrm{N})$ & $979 \pm 234$ & 4.5 & 0.8 & 8.0 & 7.2 & 0.71 & 0.78 \\
\hline $\mathrm{V}_{\max }(\mathrm{m} / \mathrm{s})$ & $1.48 \pm 0.34$ & 6.9 & 6.1 & 16.4 & 11.2 & 0.57 & 0.80 \\
\hline $\mathrm{F}_{\max } / \mathrm{F}_{\max }$ slope) & $-674 \pm 154$ & 3.2 & -0.2 & 24.8 & 23.6 & 0.35 & 0.54 \\
\hline $\mathrm{P}_{\max }(\mathrm{W})(\mathrm{P}-l$ slope $)$ & $376 \pm 150$ & 9.7 & 4.0 & 7.9 & 9.1 & 0.96 & 0.94 \\
\hline $\mathrm{P}_{\max }(\mathrm{W})(\mathrm{P}-v$ slope $)$ & $367 \pm 154$ & 13.5 & 5.6 & 12.2 & 6.1 & 0.91 & 0.97 \\
\hline Optimal load atP $\mathrm{Pax}_{\max }(\% \mathrm{BW})$ & $87.6 \pm 14.6$ & 2.4 & -1.0 & 13.7 & 10.2 & 0.26 & 0.61 \\
\hline $\begin{array}{l}\text { Optimal velocity at } P_{\max } \\
(\mathrm{m} / \mathrm{s})\end{array}$ & $0.60 \pm 0.11$ & 9.2 & 5.6 & 13.6 & 6.7 & 0.48 & 0.79 \\
\hline
\end{tabular}

Note: All variables are in the vertical direction.

SEM, standard of error of measurement represented as a CV\%; $1 \mathrm{RM}$, estimated one repetition maximal; $\mathrm{F}_{\max }$, estimated maximal force from force-velocity relationship;

$\mathrm{V}_{\max }$, maximal velocity from force-velocity relationship; $\mathrm{F}_{\max } / \mathrm{V}_{\max }$ slope, ratio between $\mathrm{F}_{\max }$ and $\mathrm{V}_{\max } ; \mathrm{P}_{\max }$, maximal power estimation using power-load $(P-l$ slope $)$ or power-velocity $(P-v$ slope $)$

${ }^{\mathrm{a}} 90 \%$ CL: $\pm 0.40 \times$ error of measurement $= \pm$ (error of measurement $) \times(\sqrt{2} / \sqrt{\mathrm{n}}) \times t$; where $n$ is the sample size, and $t$ is the $t$ statistic for $90 \%$ CL.

${ }^{b} 90 \%$ CL: $\div \times 1.20$

${ }^{\mathrm{c}} \mathrm{CL}$ : lowest ICC \pm 0.26 , highest ICC \pm 0.02 .

It was observed that predicted $1 \mathrm{RM}$ at $0.23 \mathrm{~m} / \mathrm{s}$ was reliable and unaffected by biological variation on the contrary to the study of Cronin et al. (2004), which demonstrated a significant improvement in maximal strength by as much as $15 \%$ from the first to the fourth 1RM testing occasion in novice weight trainers. The prediction based on load-velocity relationship therefore seemed to be less affected by learning compared to regular 1RM testing. The 1RM at Om/s was 30\% higher compared to the dynamic 1RM at $0.23 \mathrm{~m} / \mathrm{s}$ (Figure 2), which was in accordance with the knowledge of greater maximum isometric force compared to maximum concentric force based on the force-velocity relationship (Enoka, 2002). Jidovtseff et al. (2011) reported a smaller difference between estimated $1 \mathrm{RM}$ at $0 \mathrm{~m} / \mathrm{s}$ from the load-velocity relationship and actual $1 \mathrm{RM}(16 \%)$ in comparison to this study (30\%). Given the results of our pilot study, estimated $1 \mathrm{RM}$ at $0.23 \mathrm{~m} / \mathrm{s}$ could underestimate actual $1 \mathrm{RM}$ by $5 \%$ in the current population, which could explain partly the difference between the two studies as Jidovtseff et al. (2011) used completed 1RM as a reference. The type of population tested (adults vs. children) and the difference in the movement performed (bench press vs. supine squat machine) may have also affected the loadvelocity slope, possibly resulting in greater differences between $1 \mathrm{RM}$ at 0 and $0.23 \mathrm{~m} / \mathrm{s}$ in the current study. The reader must be mindful of these limitations when using estimated $1 \mathrm{RM}$ and should establish the velocity at $1 \mathrm{RM}$ with the specific test used. However, the high correlation $(r=0.94)$ and minimal absolute difference $(5 \%)$ between estimated $1 \mathrm{RM}$ at $0.23 \mathrm{~m} / \mathrm{s}$ and true $1 \mathrm{RM}$ in our pilot study would suggest that the estimated 1RM is a representative measure of maximal strength. In addition, considering its reliability as well as the multiple diagnostic benefits of the incremental loading protocol, such an approach is an intuitively appealing strategy for the assessment and monitoring of young athletes.

The mechanical limits of skeletal muscles' contractile elements are represented by an inverse linear forcevelocity relationship, which account for the decreased capability of the lower extremities to generate force with increasing movement velocity (Cormie et al., 2010a; Hill, 1938). Such a relationship was evidenced in the youth population investigated in this study (Figure 3). Maximal force and maximal velocity derived from this relationship were found reliable on testing sessions 2-3, but maximal velocity was likely to be influenced by familiarization and considered unreliable from sessions 1 to 2 (i.e. biological variation). Given the importance of these two fundamental mechanical entities for jumping (Samozino, Morin, Hintzy, \& Belli, 2008, 2010), a minimum of one familiarization is recommended.

The force-velocity mechanical profile of the lower limbs can be represented by the slope of maximal 
force/velocity with the force graphically represented on the vertical axis of the force-velocity relationship (Figure 3). The steeper the force-velocity relationship, the more force dominant the athlete is (Samozino et al., 2012). Due to the variability of average force and velocity and subsequent error of maximal velocity and maximal force estimation, the slope appeared unreliable. Therefore, the application of this ratio needs to be used with caution considering the large variability associated with it. It is possible that direct measurement of force (e.g. force plate) and velocity (e.g. tachometer) would reduce the variability associated with the slope (i.e. noise and mathematical estimation). This study was the first to report the reliability of this measure and therefore further investigations regarding the reliability and utility of this measurement in youth is warranted.

The maximal power achieved during ballistic movement across a load spectrum and associated recommendations for optimal loading have been widely investigated in the literature (Cormie et al., 2011). Only reporting the actual load lifted at maximal power may be misleading and recent studies have fitted quadratic equations to the power-load curve to estimate maximal power and optimal load during multi-joint exercises (mean $R^{2}=0.75$ 0.94) (Cronin et al., 2007; Harris et al., 2007). The current study found a similar goodness-of-fit of the powerload curve $\left(R^{2}=0.81\right)$. Maximal power estimation was thought reliable and the effect of a familiarization session was deemed minimal. In contrast to the findings of Harris et al. $(2007)(\mathrm{CV}=5.7-6.2 \%$; ICC $=0.86-0.92)$, the optimal load that maximized power output was found unreliable across all testing occasions. The difference between studies may be explained by the movement and loading parameters being different. Also, the reliability statistics reported by Harris et al. (2007) was only conducted within a single session, which only provides minimal insight in data variability. The lack of reliability in the optimal load that maximized power output in this study was most likely explained by the relative low biological variance in power output across the loading spectrum where a $10 \%$ and $20 \%$ change either side of the optimal load corresponded to a $1-6 \%$ change in maximal power. Both Harris et al. (2007) and Cronin et al. (2007) found a similar variance around maximal power (1-7\%) with a $10 \%$ and $20 \%$ change in optimal load, which demonstrates that load spectrum rather than specific load can be targeted for maximal power output, regardless of the type of assessment or population.

Using the group means, $88 \%$ body mass represented the optimal load for maximal power, and was equal to $41 \%$ estimated 1RM. Considering that $50 \%$ of the participants theoretical load was beyond the load spectrum $(<80 \%$ body mass), the estimated optimal load in the current study was in accordance with bench press optimal load (30-45\% 1RM) where only the external load is projected (Cormie et al., 2011). The later point is imperative when the relationship between the supine leg press squat jump power and regular squat jump power output is considered. On one hand, during a traditional squat jump, not all body parts are similarly accelerated (e.g. the lower legs are less accelerated than the upper body) and thus the mean accelerated mass and corresponding resistance force are less than body mass and vary throughout the acceleration phase. On the other hand, under the simulated $100 \%$ body mass in the supine leg press squat jump, the subject accelerates $100 \%$ of body mass in the form of the weighted plates in addition to the body mass and sled, which make the total force required above regular body mass. This limitation may explain partly why optimal load was below body mass on the supine squat machine, whereas the optimal load to maximize power output during a regular squat jump is considered to be body mass (Cormie et al., 2011). From a validity perspective, the relationship between peak power obtained during a vertical squat jump and the supine squat jump at $100 \%$ body mass load with the current participants in a pilot study was 0.89 (0.83-0.94, $90 \%$ confidence limit) and would support the utilization of the supine leg press in novice participants (Yamauchi \& Ishii, 2007).

With the accessibility of inertial dynamometers (linear position transducer or accelerometer), training at a specific velocity or optimal velocity to target maximal power has become common practice in high performance sport (Randell, Cronin, Keogh, \& Gill, 2010; Randell, Cronin, Keogh, Gill, \& Pedersen, 2011). We found that estimating maximal power based on the power-velocity curve was reliable but very likely to most likely to benefit from a familiarization session. Mean goodness-of-fit from the curve $\left\{R^{2}=0.84\right.$ ) was similar to previous findings (Rahmani et al., 2001; Samozino et al., 2012; Yamauchi \& Ishii, 2007) $\left\{R^{2}=0.70-0.99\right)$. Therefore, using the power-velocity curve to estimate maximal power and the optimal velocity to achieve maximal power is appealing. From an applied perspective, Randall et al. (2011) demonstrated the benefit of instantaneous feedback on achieving pre-specified threshold velocities for power training. Therefore, using optimal velocity as biofeedback during sessions is one manner in which the sport scientist can optimize training design and adaptations.

\section{CONCLUSIONS}

The intersession kinematics and kinetics of a ballistic loading protocol using an instrumented (linear position transducer) supine squat machine were found reliable in a youth population. Considering the unconventional nature of performing such movements in a horizontal position as well as explosively overcoming resistance greater or lower than body mass, a familiarization session was likely to provide more reliable data by reducing 
biological variation and should be conducted across the entire load spectrum. The strong load-velocity relationship appeared to be an excellent alternative to standard $1 \mathrm{RM}$ testing, and could be a preferred method to determine maximal strength for untrained or youth populations. Furthermore, the ballistic loading protocol provided additional information about the neuromuscular characteristics of the young athlete. The force-velocity relationship provides a useful tool to better explore force and velocity specificity adaptation resulting from different loading and subsequent training velocities. Also, both the power-load and power-velocity relationships can be calculated relatively quickly and reliably, and can be used to predict maximal power as well as informing loading parameters and movement velocity in training programs aiming at enhancing power output. Since growth and maturation are likely to affect both maximal force and velocity of movement, future research should investigate the influence of maturity as well as the efficacy of different training programs on the force-velocitypower profile of youth during isoinertial ballistic movement.

\section{REFERENCES}

[1] Cormie, P., McGuigan, M. R., \& Newton, R. U. (2010a). Adaptations in athletic performance after ballistic power versus strength training. Medicine \& Science in Sports \& Exercise, 42, 1582-1598.

[2] Cormie, P., McGuigan, M. R., \& Newton, R. U. (2010b). Influence of strength on magnitude and mechanisms of adaptation to power training. Medicine \& Science in Sports \& Exercise, 42, 1566-1581.

[3] Cormie, P., McGuigan, M. R., \& Newton, R. U. (2011). Developing maximal neuromuscular power. Part 2.Training considerations for improving maximal power production. Sports Medicine, 41, 125-146.

[4] Cronin, J. B., \& Henderson, M. E. (2004). Maximal strength and power assessment in novice weight trainers. Journal of Strength and Conditioning Research, 18, 48-52.

[5] Cronin, J. B., Hing, R. D., \& McNair, P. J. (2004). Reliability and validity of a linear position transducer for measuring jump performance. Journal of Strength and Conditioning Research, 18, 590593.

[6] Cronin, J. B., Jones, J. V., \& Hagstrom, J. T. (2007). Kinematics and kinetics of the seated row and implications for conditioning. Journal of Strength and Conditioning Research, 21, 1265-1270.

[7] Dayne, A. M., McBride, J. M., Nuzzo, J. L., Triplett, N. T., Skinner, J., \& Burr, A. (2011). Power output in the jump squat in adolescent male athletes. Journal of Strength and Conditioning Research, $25,585-589$.

[8] Dore, E., Duché, P., Rouffet, D., Ratel, S., Bedu, M., \& Van Praagh, E. (2003). Measurement error in short-term power testing in young people. Journal of Sports Sciences, 21, 135-142.

[9] Enoka, R. (2002). Neuromechanics of human movement. Champaign, IL: Human Kinetics.

[10]Faigenbaum, A. D., Kraemer, W. J., Blimkie, C. J., Jeffreys, I., Micheli, L. J., Nitka, M., \& Rowland, T. W. (2009).Youth resistance training: Updated position statement paper from the national strength and conditioning association. Journal of Strength and Conditioning Research, 23, S60-S79.

[11]Faigenbaum, A. D., Milliken, L., \& Wescott, W. (2004). Maximal strength testing in healthy children. Journal of Strength and Conditioning Research, 17, 162-166.

[12]Fenn, W. O., \& Marsh, B. S. (1935). Muscle force at different speed of shortening. Journal of Physiology, 85, 277-297.

[13] Harris, N. K, Cronin, J., Taylor, K. -L., \& Jidovtseff, B. (2010). Understanding position transducer technology for strength and conditioning practitioners. Strength and Conditioning Journal, 32, 66-79.

[14] Harris, N. K, Cronin, J. B., \& Hopkins, W. G (2007). Power outputs of a machine squat-jump across a spectrum of loads. Journal of Strength and Conditioning Research, 21, 1260-1264.

[15] Hill, A. V. (1938). The heat of shortening and the dynamic constant of muscle. Proceedings of the Royal Society of London, Series B, Biological Sciences, 126, 136-195.

[16] Hopkins, W. G (2000). Measures of reliability in sports medicine and science. Sports Medicine, 30, 115.

[17] Hopkins, W. G., \& Hewson, D. J. (2001). Variability of competitive performance of distance runners. Medicine \& Science in Sports \& Exercise, 33, 1588-1592.

[18]Hopkins, W. G., Marshall, S. W., Batterham, A. M., \& Hanin, J. (2009). Progressive statistics for 
studies in sports medicine and exercise science. Medicine \& Science in Sports \& Exercise, 41, 3-13.

[19] Hori, N., Newton, R. U., Andrews, W. A., Kawamori, N., McGuigan, M. R., \& Nosaka, K. (2007). Comparison of four different methods to measure power output during the hang power clean and the weighted jump squat. Journal of Strength and Conditioning Research, 21, 314-320.

[20] Jidovtseff, B., Harris, N. K., Crielaard, J., \& Cronin, J. B. (2011). Using the load-velocity relationship for 1RM prediction. Journal of Strength and Conditioning Research, 25, 267-270.

[21] Khamis, H. J., \& Roche, A. F. (1994). Predicting adult stature without using skeletal age: The KhamisRoche method. Pediatrics, 94, 504-507.

[22] Mirwald, R. L., Baxter-Jones, A. D., Bailey, D. A., \& Beunen, G. P. (2002). An assessment of maturity from anthropometric measurements. Medicine \& Science in Sports \& Exercise, 34, 689-694.

[23] Rahmani, A., Viale, R, Dalleau, G., \& Lacour, J. R. (2001). Force/velocity and power/velocity relationships in squat exercise. European Journal of Applied Physiology, 84, 227-232.

[24] Randell, A. D., Cronin, J., Keogh, J. W., \& Gill, N. D. (2010). Optimizing within session training emphasis. Journal of Strength and Conditioning Research, 32, 73-80.

[25] Randell, A. D., Cronin, J. B., Keogh, J. W., Gill, N. D., \& Pedersen, M. C. (2011). Effect of instantaneous performance feedback during 6 weeks of velocity-based resistance training on sportspecific performance tests. Journal of Strength and Conditioning Research, 25, 87-93.

[26] Samozino, P., Morin, J. B., Hintzy, F., \& Belli, A. (2008). A simple method for measuring force, velocity and power output during squat jump. Journal of Biomechanics, 41, 2940-2945.

[27] Samozino, P., Morin, J. B., Hintzy, F., \& Belli, A. (2010). Jumping ability: A theoretical integrative approach. Journal of Theoretical Biology, 2 64, 11-18.

[28] Samozino, P., Rejc, E., Di Prampero, P. E., Belli, A., \& Morin, J. B. (2012). Optimal force-velocity profile in ballistic movements - altius: Citius or fortius? Medicine and Science in Sports and Exercise, 44, 313-322.

[29] Santos, A., Welsman, J. R., De Ste Croix, M., \& Armstrong, N. (2002). Age- and sex-related differences in optimal peak power. Pediatric Exercise Science, 14, 202-212.

[30] Sheppard, J. M., Cormack, S., Taylor, K. L., McGuigan, M. R., \& Newton, R. U. (2008). Assessing the force-velocity characteristics of the leg extensors in well-trained athletes: The incremental load power profile. Journal of Strength and Conditioning Research, 22, 1320-1326.

[31] Stokes, M. (1985). Reliability and repeatability of methods for measuring muscle in physiotherapy. Physiotherapy Practice, 1, 71-76.

[32] Van Praagh, E. (2008). Testing anaerobic performance. In H. Hebestreit \& O Bar-Or (Eds.), The young athlete (pp. 453-468). Maiden: Blackwell.

[33] Van Praagh, E., \& Dore, E. (2002). Short-term muscle power during growth and maturation. Sports Medicine, 32, 701-728.

[34] Viitasalo, J. (1988). Evaluation of explosive strength for young and adult athletes. Research Quarterly, 59, 9-13.

[35] Viitasalo, J., Rahkila, P., Osterback, L., \& Alen, M. (1992). Vertical jumping height and horizontal overhead throwing velocity in young male athletes. Journal of Sports Sciences, 10, 401-413.

[36] Vincent, J. (1994). Statistics in kinesiology. Champaign, IL: Human Kinetics.

[37] Yamauchi, J., \& Ishii, N. (2007). Relations between force-velocity characteristics of the knee-hip extension movement and vertical jump performance. Journal of Strength and Conditioning Research, 21, 703-709. 